Supporting Information (5 pages)

\title{
Synthesis of Allenes via Palladium-Catalyzed Hydrogen Transfer Reactions: Propargylic Amines As an Allenyl Anion Equivalent
}

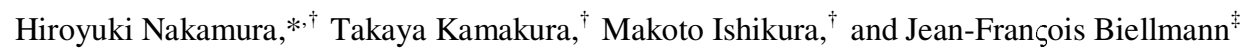 \\ ${ }^{\dagger}$ Department of Chemistry, Faculty of Science, Gakushuin University, Mejiro, Toshima-ku, Tokyo 171-8588, Japan \\ ${ }^{+}$Institute of Chemistry, Academia Sinica, Nankang Taipei, Taiwan
}

\begin{abstract}
General Information. ${ }^{1} \mathrm{H}$ and ${ }^{13} \mathrm{C}$ NMR spectra were recorded on a JEOL JNM-AL 300 (300 MHz) or a VARIAN UNITY-INOVA $400(400 \quad \mathrm{MHz})$ spectrometers. The chemical shifts are reported in $\delta$ units relative to internal tetramethylsilane. IR spectra were recorded on a Shimadzu FTIR-8200A spectrometer. High-resolution mass spectra (EI and FAB) were recorded on a Jeol JMS-HX110. Most commercially supplied chemicals were used without further purification. The structure of phenylallene 4a was determined unambiguously by comparison with authentic sample prepared by the literature procedure. ${ }^{1,2}$
\end{abstract}

Hydrogen transfer reactions of 3ad under various palladium-catalyzed conditions and plausible mechanisms.

Hydrogen transfer reactions of $\mathbf{3 a d}$ proceeded in the presence of $\mathrm{Pd}(0)$ catalysts, such as $\mathrm{Pd}_{2} \mathrm{dba}_{3} \cdot \mathrm{CHCl}_{3}$ and $\mathrm{Pd}_{2} \mathrm{dba}_{3}$ $(2.5 \mathrm{~mol} \%)$, with $\left(\mathrm{C}_{6} \mathrm{~F}_{5}\right)_{3} \mathrm{P}(20 \mathrm{~mol} \%)$ to give $4 \mathrm{a}$ in $99 \%$ yields (entries 1 and 2 in Table 1$)$. In the absence of $\left(\mathrm{C}_{6} \mathrm{~F}_{5}\right)_{3} \mathrm{P}$ as a ligand, $\mathbf{4 a}$ was obtained in $5 \%$ yield (entry 3). A referee pointed out the possibility of Pd (II) as an actual catalyst. We examined the hydrogen transfer reaction of $\mathbf{3 a d}$ using $\mathrm{Pd}(\mathrm{OAc})_{2} /\left(\mathrm{C}_{6} \mathrm{~F}_{5}\right)_{3} \mathrm{P}$ as catalysts and $\mathbf{4 a}$ was obtained in $95 \%$ yield (entry 4). Even in the absence of the ligand, the reaction proceeded to give $4 \mathbf{a}$ in $61 \%$ yield along with the recovery of $\mathbf{3 a d}$ in $31 \%$ yield (entry 5 ). Since the oxidative insertion of Pd (0) to a C-H bond adjacent to nitrogen was investigated by Murahashi and the coworkers, ${ }^{3}$ we may propose a $\operatorname{Pd}(0)$ mechanism as shown in Scheme 1. Oxidative insertion of $\mathrm{Pd}(0)$ to $\mathbf{3}$ would form the $\mathrm{Pd}$ (II) complex $\mathbf{7}$, which comes to rapid equilibrium with the iminium ion complex $\mathbf{8}$ assisted by a lone pair electron of the nitrogen. The hydrogen transfer from palladium to alkyne moiety of $\mathbf{8}$ followed by the rearrangement of a $\pi$-bond would give the allenes 4 and the imine 9, and palladium $(0)$ is regenerated. Since $\left(\mathrm{C}_{6} \mathrm{~F}_{5}\right)_{3} \mathrm{P}$ is an electron-deficient phosphine and an effective ligand for the $\operatorname{Pd}(0)$-catalyzed reaction, it would be considered that a role of $\left(\mathrm{C}_{6} \mathrm{~F}_{5}\right)_{3} \mathrm{P}$ is the stabilization of the anionic palladium intermediate 8 in the equilibrium between 7 and $\mathbf{8}$ to accelerate the generation of allenes $\mathbf{4}$ in the catalytic cycle (Scheme 1). Although the imine $\mathbf{9}$ was not detected in the reaction mixture, benzyl benzylideneamine was detected by GC-MS in the case of the reaction of 3ab. This result strongly supports our proposed mechanism. According to the result obtained from entry 4 in Table 1, an alternative mechanism, in which Pd (II) would be a reactive intermediate through the catalytic cycle, could be envisaged as shown in Scheme 2. The coordination of $\operatorname{Pd}(\mathrm{OAc})_{2}$ with $\mathbf{3}$ would form the complex 7', which comes to rapid equilibrium with the iminium ion complex $\mathbf{8}$, assisted by a lone pair electron of the nitrogen. The hydrogen transfer from H-Pd-OAc to alkyne moiety of $\mathbf{8}$ followed by the rearrangement of $\pi$-bond would give the allenes $\mathbf{4}$ and the imine $\mathbf{9}$, and $\mathrm{Pd}(\mathrm{OA})_{2}$ is regenerated. 
Table 1. Hydrogen transfer reactions of 3ad under various palladium-catalyzed conditions ${ }^{a}$

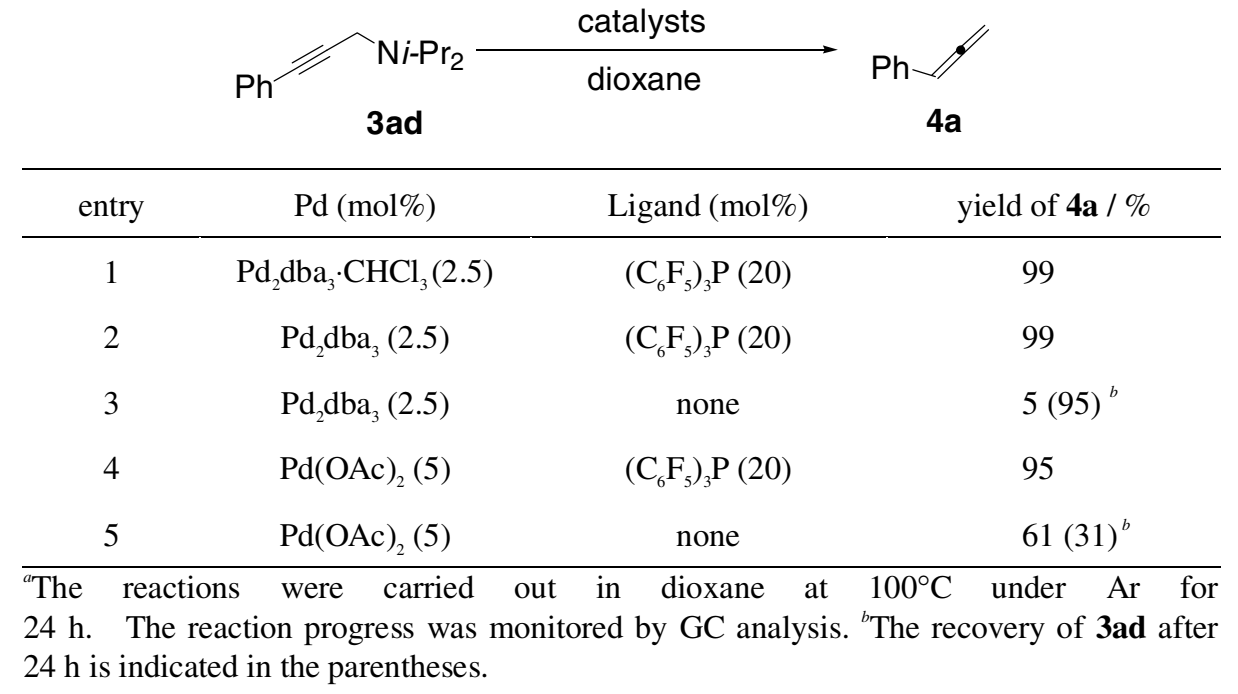

Scheme 1. The $\operatorname{Pd}(0)$ Mechanism

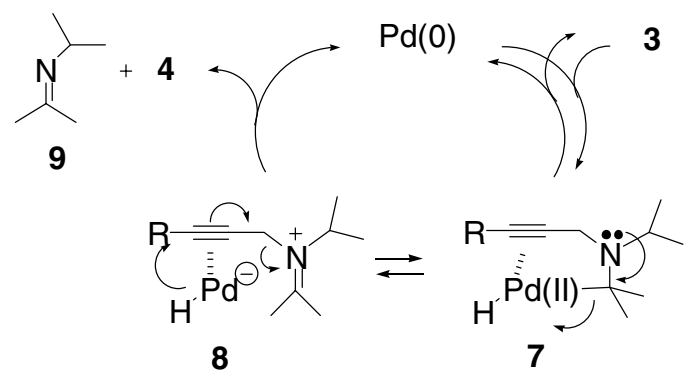

Scheme 2. The Pd(II) Mechanism

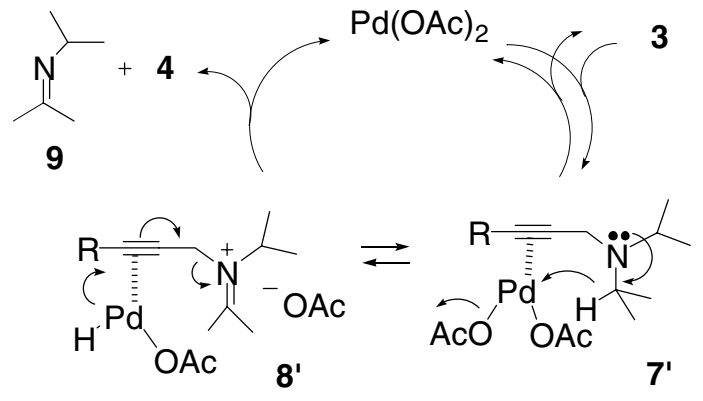

\section{Typical procedure for the synthesis of propargylamines by Sonogashira coupling reactions.}

A mixture of 1a $(205 \mathrm{mg}, 1.0 \mathrm{mmol}), \mathrm{Pd}\left(\mathrm{PPh}_{3}\right)_{4}(35 \mathrm{mg}, 0.03 \mathrm{mmol}), \mathrm{CuI}(17 \mathrm{mg}, 0.09 \mathrm{mmol})$, and $2 d$ (180 mg, 1.3 mmol) was dissolved in acetonitril $(5 \mathrm{~mL})$ under Ar and triethylamine $(210 \mu \mathrm{L}, 1.5 \mathrm{mmol})$ was added. The mixture was stirred at $60{ }^{\circ} \mathrm{C}$ for $6 \mathrm{~h}$. The reaction progress was monitored by GC. The solvent was removed under the reduced pressure and the residue was purified by silica gel column chromatography with hexane/ethyl acetate (10/1) to give 3ad (185 mg, 86\% yield). 
$\boldsymbol{N}, \boldsymbol{N}$-diethyl-3-phenylprop-2-ynylamine (3aa). Colorless oil; ${ }^{1} \mathrm{H}$ NMR (400 MHz, $\left.\mathrm{CDCl}_{3}\right) \delta 7.42-7.39$ (m, 2H), 7.28-7.26 (m, 2H), $3.63(\mathrm{~s}, 2 \mathrm{H}), 2.61(\mathrm{q}, J=7.2 \mathrm{~Hz}, 4 \mathrm{H}), 1.10(\mathrm{t}, J=7.2 \mathrm{~Hz}, 6 \mathrm{H}) ;{ }^{13} \mathrm{C} \mathrm{NMR}\left(75 \mathrm{MHz}, \mathrm{CDCl}_{3}\right) \delta 131.6$, 128.1, 127.6, 123.2, 84.9, 84.3, 47.3, 41.5, 12.8; IR 2970, 1599, 1489, 1443, 1200, 1092, $1069 \mathrm{~cm}^{-1}$; HRMS (FAB) calcd for $\mathrm{C}_{13} \mathrm{H}_{18} \mathrm{~N}:(\mathrm{M}+\mathrm{H})^{+} \mathrm{m} / \mathrm{z}$ 188.1439, found $\mathrm{m} / \mathrm{z} 188.1434$.

$N, N$-dibenzyl-3-phenylprop-2-ynylamine (3ab). White solid; ' $\mathrm{H}$ NMR (400 $\left.\mathrm{MHz}, \mathrm{CDCl}_{3}\right) \delta 7.50 \sim 7.47$ (m, $2 \mathrm{H}$ ), 7.43 7.41 (m, 3H), 7.34 7.30 (m, 6H), 7.27 7.22 (m, 4H), $3.74(\mathrm{~s}, 4 \mathrm{H}), 3.46(\mathrm{~s}, 2 \mathrm{H}) ;{ }^{13} \mathrm{C}$ NMR $\left(75 \mathrm{MHz}, \mathrm{CDCl}_{3}\right)$ $\delta 138.8,131.7,129.0,128.2,127.9,127.0,85.8,84.4,57.7,42.1 ; \mathrm{IR} 3028,2826,1597,1489,1454,918 \mathrm{~cm}^{-1}$; HRMS (FAB) calcd for $\mathrm{C}_{23} \mathrm{H}_{22} \mathrm{~N}$ : $(\mathrm{M}+\mathrm{H})^{+} \mathrm{m} / \mathrm{z}$ 312.1752, found $\mathrm{m} / \mathrm{z}$ 312.1747. Calcd for $\mathrm{C}_{23} \mathrm{H}_{21} \mathrm{NNa}:(\mathrm{M}+\mathrm{Na})^{+} \mathrm{m} / \mathrm{z}$ 334.1572, found $\mathrm{m} / \mathrm{z} 334.1566$.

$N, N$-diisobutylprop-3-phenylprop-2-ynylamine (3ac). Colorless oil; ' ${ }^{\mathrm{H}} \mathrm{NMR}\left(400 \mathrm{MHz}, \mathrm{CDCl}_{3}\right) \delta 7.43-7.41$ (m, 2H), 7.31-7.26 (m, 3H), 3.55 (s, 2H), $2.26(\mathrm{~d}, J=7.2 \mathrm{~Hz}, 4 \mathrm{H}), 1.73$, (tsept, $J=7.2,6.4 \mathrm{~Hz}, 2 \mathrm{H}), 0.90$ (d, $J=6.4 \mathrm{~Hz}$, $12 \mathrm{H}) ;{ }^{13} \mathrm{C}$ NMR $\left(75 \mathrm{MHz}, \mathrm{CDCl}_{3}\right) \delta 131.5,128.0,127.6,123.5,85.4,84.6,62.7,43.5,26.4,20.9 ;$ IR 2953, 2868, 1597 , 1489, 1468, 1364, $1072 \mathrm{~cm}^{-1}$; HRMS (FAB) calcd for $\mathrm{C}_{17} \mathrm{H}_{26} \mathrm{~N}:(\mathrm{M}+\mathrm{H})^{+} \mathrm{m} / z$ 244.2065, found $\mathrm{m} / z$ 244.2060.

$\mathrm{N}, \mathrm{N}$-diisopropyl-3-phenylprop-2-ynylamine (3ad). Colorless oil; ${ }^{1} \mathrm{H}$ NMR (400 MHz, $\left.\mathrm{CDCl}_{3}\right) \delta$ 7.41-7.38 (m, $\left.2 \mathrm{H}\right)$, 7.28-7.26 (m, 3H), 3.65 (s, 2H), 3.26 (sept, $J=6.4 \mathrm{~Hz}, 2 \mathrm{H}), 1.15(\mathrm{~d}, J=6.4 \mathrm{~Hz}, 12 \mathrm{H}){ }^{13} \mathrm{C}$ NMR $\left(75 \mathrm{MHz}, \mathrm{CDCl}_{3}\right) \delta$ 131.2, 128.0, 127.5, 123.6, 88.9, 83.4, 48.5, 34.8, 20.7; IR 2966, 1598, 1488, 1380, 1203, $1176 \mathrm{~cm}^{-1}$; HRMS (FAB) calcd for $\mathrm{C}_{15} \mathrm{H}_{22} \mathrm{~N}:(\mathrm{M}+\mathrm{H})^{+} \mathrm{m} / z, 216.1752$, found $\mathrm{m} / z 216.1747$.

cis-2,6-dimethyl-1-(3-phenyl-prop-2-ynyl)-piperidine (3ae). Yellow oil; ' $\mathrm{H}$ NMR (400 MHz, $\left.\mathrm{CDCl}_{3}\right) \delta$ 7.45-7.43, $(\mathrm{m}, 2 \mathrm{H}), 7.32-7.29(\mathrm{~m}, 3 \mathrm{H}), 3.87(\mathrm{~s}, 2 \mathrm{H}), 2.61-2.58(\mathrm{~m}, 2 \mathrm{H}), 1.69-1.62(\mathrm{~m}, 4 \mathrm{H}), 1.42-1.29(\mathrm{~m}, 2 \mathrm{H}), 1.17(\mathrm{~d}, J=\mathrm{Hz}$, $2 \mathrm{H}) ;{ }^{13} \mathrm{C}$ NMR (75 MHz, $\mathrm{CDCl}_{3}$ ) $\delta 131.6,128.1,127.7,123.3,85.0,83.5,54.8,38.1,35.4,24.8,21.1$; IR 1926, 1489, 1441, 1317, 1200, 1094, $1059 \mathrm{~cm}^{-1}$; HRMS $(\mathrm{FAB})$ calcd for $\mathrm{C}_{16} \mathrm{H}_{22} \mathrm{~N}:(\mathrm{M}+\mathrm{H})^{+} \mathrm{m} / \mathrm{z}$ 228.1752, found $\mathrm{m} / \mathrm{z}$ 228.1747. Calcd for $\mathrm{C}_{16} \mathrm{H}_{21} \mathrm{NNa}(\mathrm{M}+\mathrm{Na})^{+} \mathrm{m} / 2$ 250.1572, found $\mathrm{m} / z 250.1566$.

$\boldsymbol{N}, \boldsymbol{N}$-diisopropyl-3-(3-naphthyl)prop-2-ynylamine (3bd). Colorless oil; ${ }^{1} \mathrm{H} \mathrm{NMR}\left(400 \mathrm{MHz}, \mathrm{CDCl}_{3}\right) \delta 8.33$ (d, $J=$ $8.4 \mathrm{~Hz}, 1 \mathrm{H}$ ), 7.79 (dd, $J=8.4,7.2 \mathrm{~Hz}, 2 \mathrm{H}), 7.62$ (d, $J=7.2 \mathrm{~Hz}, 1 \mathrm{H}), 7.56-7.37$ (m, 3H), 3.83 (s, 2H), 3.34 (sept, $J=$ $6.8 \mathrm{~Hz}, 2 \mathrm{H}), 1.21(\mathrm{~d}, J=6.8 \mathrm{~Hz}, 12 \mathrm{H}) ;{ }^{13} \mathrm{C}$ NMR $\left(75 \mathrm{MHz}, \mathrm{CDCl}_{3}\right) \delta 133.3,133.0,130.0,128.0,127.9,126.4,126.0$, 125.0, 121.3, 93.8, 81.6, 48.5, 35.0, 20.8; IR 3057, 2968, 1508, 1458, 1380, 1203, $1176 \mathrm{~cm}^{-1}$; HRMS (FAB) calcd for $\mathrm{C}_{19} \mathrm{H}_{24} \mathrm{~N}:(\mathrm{M}+\mathrm{H})^{+} \mathrm{m} / z$ 266.1906, found $\mathrm{m} / z$ 266.1903. Calcd for $\mathrm{C}_{19} \mathrm{H}_{23} \mathrm{NNa}$ : (M+Na) ${ }^{+} \mathrm{m} / z, 288.1728$, found $\mathrm{m} / z$ 288.1723.

$N, N$-diisopropyl-3-(4-methoxypheyl)-prop-2-ynylamine (3cd). Colorless oil; ${ }^{1} \mathrm{H}$ NMR (400 MHz, $\left.\mathrm{CDCl}_{3}\right) \delta 7.33$ (d, $J$ $=8.8 \mathrm{~Hz}, 2 \mathrm{H}), 6.81,(\mathrm{~d}, J=8.8 \mathrm{~Hz}, 2 \mathrm{H}), 3.78(\mathrm{~s}, 3 \mathrm{H}), 3.64(\mathrm{~s}, 2 \mathrm{H}), 3.26(\mathrm{sept}, J=6.8 \mathrm{~Hz}, 2 \mathrm{H}), 1.14(\mathrm{~d}, J=6.8 \mathrm{~Hz}, 12 \mathrm{H})$; ${ }^{13} \mathrm{C}$ NMR $\left(75 \mathrm{MHz}, \mathrm{CDCl}_{3}\right) \delta 158.9,132.6,115.8,113.6,87.3,83.1,55.2,48.4,34.8,20.7$; IR 2966, 1606, 1508, 1463 , 1290, 1247, 1176, $1035 \mathrm{~cm}^{-1}$; HRMS (FAB) calcd for $\mathrm{C}_{16} \mathrm{H}_{24} \mathrm{NO}:(\mathrm{M}+\mathrm{H})^{+} \mathrm{m} / \mathrm{z}$ 246.1858, found $\mathrm{m} / \mathrm{z}$ 246.1852. Calcd for $\mathrm{C}_{16} \mathrm{H}_{23} \mathrm{NONa}(\mathrm{M}+\mathrm{Na})^{+} \mathrm{m} / \mathrm{z} 268.1677$, found $\mathrm{m} / z 268.1672$.

$N$-4-(3- $N, N$-diisopropylaminoprop-1-ynyl)-phenylacetamide (3dd). White solid; ${ }^{1} \mathrm{H}$ NMR (400 MHz, $\left.\mathrm{CDCl}_{3}\right) \delta 7.90$ (bs, 1H), 7.45 (d, $J=8.4 \mathrm{~Hz}, 2 \mathrm{H}), 7.32$ (d, $J=8.4 \mathrm{~Hz}, 2 \mathrm{H}), 3.63$ (s, 2H), 3.25 (sept, $J=6.4 \mathrm{~Hz}, 2 \mathrm{H}), 2.15$ (s, 3H), 1.14 $(\mathrm{d}, J=6.4 \mathrm{~Hz}, 12 \mathrm{H}) ;{ }^{13} \mathrm{C} \mathrm{NMR}\left(75 \mathrm{MHz}, \mathrm{CDCl}_{3}\right) \delta 168.3,137.3,131.9,119.3,119.2,88.4,83.0,48.5,34.8,24.6$, 
20.7; IR 3257, 2972, 1664, 1600, 1510, 1373, 1203, 1178, 1029, 839 $\mathrm{cm}^{-1}$; HRMS (FAB) calcd for $\mathrm{C}_{17} \mathrm{H}_{25} \mathrm{~N}_{2} \mathrm{O}:(\mathrm{M}+\mathrm{H})^{+}$ $m / z$ 273.1967, found $m / z$ 273.1961. Calcd for $\mathrm{C}_{17} \mathrm{H}_{24} \mathrm{~N}_{2} \mathrm{ONa}$ : $(\mathrm{M}+\mathrm{Na})^{+} \mathrm{m} / z$ 295.1786, found $\mathrm{m} / z$ 295.1781.

4-(3-N,N-diisopropylaminoprop-1-ynyl)-benzoic acid ethylester (3ed). Yellow oil; ${ }^{1} \mathrm{H} \quad \mathrm{NMR}$ (400 $\left.\mathrm{MHz}, \mathrm{CDCl}_{3}\right) \delta 7.96(\mathrm{~d}, J=8.8 \mathrm{~Hz}, 2 \mathrm{H}), 7.44(\mathrm{~d}, J=8.8 \mathrm{~Hz}, 2 \mathrm{H}), 4.36(\mathrm{q}, J=7.2 \mathrm{~Hz}, 2 \mathrm{H}), 3.66(\mathrm{~s}, 2 \mathrm{H}), 3.25$ (sept, $J=$ $6.8 \mathrm{~Hz}, 2 \mathrm{H}), 1.38(\mathrm{t}, J=7.2 \mathrm{~Hz}, 3 \mathrm{H}), 1.15(\mathrm{~d}, J=6.8 \mathrm{~Hz}, 12 \mathrm{H}){ }^{13} \mathrm{C}$ NMR $\left(75 \mathrm{MHz}, \mathrm{CDCl}_{3}\right) \delta 165.8,131.8,129.2,128.2$, 92.4, 82.8, 61.0, 48.5, 34.9, 20.7, 14.3; IR 2970, 1720, 1606, 1463, 1365, 1271, 1174, 1105, 1020, $858 \mathrm{~cm}^{-1}$; HRMS (FAB) calcd for $\mathrm{C}_{18} \mathrm{H}_{26} \mathrm{NO}_{2}:(\mathrm{M}+\mathrm{H})^{+} \mathrm{m} / \mathrm{z}$, 288.1964, found $\mathrm{m} / \mathrm{z}$ 288.1958. Calcd for $\mathrm{C}_{18} \mathrm{H}_{25} \mathrm{NO}_{2} \mathrm{Na}:(\mathrm{M}+\mathrm{Na})^{+} \mathrm{m} / z$ 310.1783, found $\mathrm{m} / \mathrm{z} 310.1778$.

$\boldsymbol{N}, \boldsymbol{N}$-diisopropyl-3-(4-nitrophenyl)-prop-2-ynylamine (3fd). Yellow oil; ${ }^{1} \mathrm{H}$ NMR $\left(400 \mathrm{MHz}, \mathrm{CDCl}_{3}\right) \delta 8.16(\mathrm{~d}, J=$ $9.2 \mathrm{~Hz}, 2 \mathrm{H}), 7.52(\mathrm{~d}, J=9.2 \mathrm{~Hz}, 2 \mathrm{H}), 3.71(\mathrm{~s}, 2 \mathrm{H}), 3.28$ (sept, $J=6.8 \mathrm{~Hz}, 2 \mathrm{H}), 1.17(\mathrm{~d}, J=6.8 \mathrm{~Hz}, 12 \mathrm{H}) ;{ }^{13} \mathrm{C}$ NMR $(75$ $\left.\mathrm{MHz}, \mathrm{CDCl}_{3}\right) \delta 146.5,131.9,130.4,123.3,94.8,82.0,48.8,34.9,20.5$; IR 2970, 2229, 1593, 1519, 1342, 1307, 1203 , 1194, 1101, $854 \mathrm{~cm}^{-1}$; HRMS (FAB) calcd for $\mathrm{C}_{15} \mathrm{H}_{21} \mathrm{~N}_{2} \mathrm{O}_{2}:(\mathrm{M}+\mathrm{H})^{+} \mathrm{m} / z, 261.1603$, found $\mathrm{m} / z, 261.1598$.

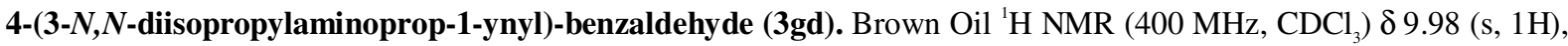
$7.80(\mathrm{~d}, J=7.2 \mathrm{~Hz}, 2 \mathrm{H}), 7.53(\mathrm{~d}, J=7.2 \mathrm{~Hz}, 2 \mathrm{H}), 3.70$ (s, 2H), 3.28 (sept, $J=6.4 \mathrm{~Hz}, 2 \mathrm{H}), 1.17$ (d, $J=6.4 \mathrm{~Hz}, 12 \mathrm{H})$; ${ }^{13} \mathrm{C}$ NMR $\left(75 \mathrm{MHz}, \mathrm{CDCl}_{3}\right) \delta$ 191.1, 134.9, 131.7, 129.8, 129.3, 93.4, 82.9, 48.7, 34.9, 20.6; IR 2970, 1703, 1602, 1560, 1263, 1301, 1207, 1164, $831 \mathrm{~cm}^{-1}$; HRMS (ESI) calcd for $\mathrm{C}_{16} \mathrm{H}_{22} \mathrm{NO}$ : $(\mathrm{M}+\mathrm{H})^{+} \mathrm{m} / \mathrm{z}, 244.1701$, found $\mathrm{m} / \mathrm{z}, 244.1696$.

1-Allenylnaphthalene (4b). White solid ${ }^{1} \mathrm{H}$ NMR $\left(400 \mathrm{MHz}, \mathrm{CDCl}_{3}\right) \delta 8.20(\mathrm{~d}, J=7.6 \mathrm{~Hz}, 1 \mathrm{H}), 7.85(\mathrm{~d}, J=7.6 \mathrm{~Hz}$, $1 \mathrm{H}), 7.74(\mathrm{~d}, J=8.4 \mathrm{~Hz}, 1 \mathrm{H}), 7.58(\mathrm{~d}, J=6.8 \mathrm{~Hz}, 1 \mathrm{H}), 7.52-7.42(\mathrm{~m}, 3 \mathrm{H}), 6.86(\mathrm{t}, J=6.8 \mathrm{~Hz}, 1 \mathrm{H}), 5.20(\mathrm{~d}, J=6.8 \mathrm{~Hz}$, $2 \mathrm{H}) ;{ }^{13} \mathrm{C} \mathrm{NMR}\left(75 \mathrm{MHz}, \mathrm{CDCl}_{3}\right) \delta 210.7,133.7,129.9,128.5,127.3,125.8,125.5,125.4,125.1,124.7,123.3,90.3$, 77.8; IR (KBr) 1940, 1649, 1494, 1392, 1301, 1232, 1101, $987 \mathrm{~cm}^{-1}$; HRMS (EI) calcd for $\mathrm{C}_{13} \mathrm{H}_{10}:\left(\mathrm{M}^{+}\right) \mathrm{m} / z$ 166.0781, found $\mathrm{m} / \mathrm{z}, 166.0777$.

1-Methoxy-4-allenylbenzene (4c). Colorless oil; ' ${ }^{\mathrm{H}} \mathrm{NMR}\left(400 \mathrm{MHz}, \mathrm{CDCl}_{3}\right) \delta 7.21(\mathrm{~d}, J=8.8 \mathrm{~Hz}, 2 \mathrm{H}), 6.84(\mathrm{~d}, J=$ $8.8 \mathrm{~Hz}, 2 \mathrm{H}), 6.11(\mathrm{t}, J=6.8 \mathrm{~Hz}, 1 \mathrm{H}), 5.11(\mathrm{~d}, J=6.8 \mathrm{~Hz}, 2 \mathrm{H}), 3.79(\mathrm{~s}, 3 \mathrm{H}) ;{ }^{13} \mathrm{C}$ NMR $\left(75 \mathrm{MHz}, \mathrm{CDCl}_{3}\right)$ $\delta 209.4,158.5,127.6,125.9,114.0,93.3,78.6,55.3 ; \quad$ IR(neat) 2837, 1942, 1608, 1487, 1247, 1087, $1035 \mathrm{~cm}^{-1} ; \quad$ MS (EI) $\mathrm{m} / \mathrm{z}, 146\left(\mathrm{M}^{+}\right), 131\left([\mathrm{M}-\mathrm{Me}]^{+}\right), 115\left([\mathrm{M}-\mathrm{OMe}]^{+}\right)$.

4-Allenylacetanilide (4d). White solid; ${ }^{1} \mathrm{H}$ NMR $\left(400 \mathrm{MHz}, \mathrm{CDCl}_{3}\right) \delta 7.57$ (bs, $\left.1 \mathrm{H}\right), 7.45(\mathrm{~d}, J=8.4 \mathrm{~Hz}, 2 \mathrm{H}), 7.23(\mathrm{~d}, J$ $=8.4 \mathrm{~Hz}, 2 \mathrm{H}), 6.12(\mathrm{t}, J=6.8 \mathrm{~Hz}, 1 \mathrm{H}), 5.13(\mathrm{~d}, J=6.8 \mathrm{~Hz}, 2 \mathrm{H}), 2.15(\mathrm{~s}, 3 \mathrm{H}) ;{ }^{13} \mathrm{C} \mathrm{NMR}\left(75 \mathrm{MHz}, \mathrm{CDCl}_{3}\right) \delta 209.7$, 168.4, 136.7, 129.8, 127.2, 120.1, 93.3, 78.9, 24.5; IR (KBr) 3057, 1944, 1660, 1602, 1508, 1404, 1369, $1263 \mathrm{~cm}^{-1}$; MS (EI) $\quad m / z, 173\left(\mathrm{M}^{+}\right), 130\left([\mathrm{M}-\mathrm{Ac}]^{+}\right), 115\left([\mathrm{M}-\mathrm{NHAc}]^{+}\right)$.

Ethyl 4-allenylbenzoate (4e). Colorless oil ${ }^{1} \mathrm{H}$ NMR $\left(400 \mathrm{MHz}, \mathrm{CDCl}_{3}\right) \delta 7.98(\mathrm{~d}, J=8.4 \mathrm{~Hz}, 2 \mathrm{H}), 7.34(\mathrm{~d}, J=8.0 \mathrm{~Hz}$, $2 \mathrm{H}), 6.19(\mathrm{~d}, J=6.8 \mathrm{~Hz}, 1 \mathrm{H}), 5.20(\mathrm{t}, J=6.8 \mathrm{~Hz}, 2 \mathrm{H}), 4.37(\mathrm{q}, J=7.2 \mathrm{~Hz}, 2 \mathrm{H}), 1.25(\mathrm{t}, J=6.0, \mathrm{~Hz}) ;{ }^{13} \mathrm{C}$ NMR $(75$ $\left.\mathrm{MHz}, \mathrm{CDCl}_{3}\right) \delta 210.6,166.4,138.8,129.8,128.7,126.4,93.6,79.1,60.8,14.2$; IR (neat) 3357, 2981, 1940, 1716, 1608 , $1517,1274 \mathrm{~cm}^{-1}$; HRMS (EI) calcd for $\mathrm{C}_{12} \mathrm{H}_{12} \mathrm{O}_{2}:\left(\mathrm{M}^{+}\right) \mathrm{m} / \mathrm{z}, 188.0837$, found $m / z, 188.0843$

4-Allenylnitrobenzene (4f). Yellow oil; ${ }^{1} \mathrm{H} \mathrm{NMR}\left(400 \mathrm{MHz}, \mathrm{CDCl}_{3}\right) \delta 8.16(\mathrm{~d}, J=8.7 \mathrm{~Hz}, 2 \mathrm{H}), 7.42(\mathrm{~d}, J=8.7 \mathrm{~Hz}, 2 \mathrm{H})$, $6.24(\mathrm{t}, \quad J=6.6 \mathrm{~Hz}, \quad 1 \mathrm{H}), \quad 5.27(\mathrm{~d}, \quad J=6.6 \mathrm{~Hz}, \quad 1 \mathrm{H}) ;{ }^{13} \mathrm{C} \quad \mathrm{NMR} \quad\left(75 \mathrm{MHz}, \quad \mathrm{CDCl}_{3}\right)$ $\delta 210.9,141.2,126.9,123.9,93.0,79.7 ; \quad$ IR (neat) $1938,1643,1595,1471,1384,1342,1139,858 \mathrm{~cm}^{-1}$; HRMS (EI) 
calcd for $\mathrm{C}_{9} \mathrm{H}_{7} \mathrm{NO}_{2}:\left(\mathrm{M}^{+}\right) \mathrm{m} / \mathrm{z}, 161.0477$, found $\mathrm{m} / \mathrm{z}, 161.0466$.

4-Allenylbenzaldehyde (4g). Colorless oil; ${ }^{1} \mathrm{H}$ NMR (400 MHz, $\left.\mathrm{CDCl}_{3}\right) \delta 9.97(\mathrm{~s}, 1 \mathrm{H}), 7.82(\mathrm{~d}, J=8.0 \mathrm{~Hz}, 2 \mathrm{H}), 7.44$ $(\mathrm{d}, J=8.0 \mathrm{~Hz}, 2 \mathrm{H}), 6.22(\mathrm{t}, J=6.8 \mathrm{~Hz}, 1 \mathrm{H}), 5.24(\mathrm{~d}, J=6.8 \mathrm{~Hz}, 2 \mathrm{H}) ;{ }^{13} \mathrm{C} \mathrm{NMR}\left(75 \mathrm{MHz}, \mathrm{CDCl}_{3}\right) \delta 210.7,191.4,140.7$, 134.8, 130.0, 126.9, 93.6, 79.4; IR (neat) 2734, 1938, 1697, 1602, 1573, 1487, 1438, $1388 \mathrm{~cm}^{-1}$; HRMS (EI) calcd for $\mathrm{C}_{15} \mathrm{H}_{14} \mathrm{~N}_{2} \mathrm{O}:\left(\mathrm{M}^{+}\right) \mathrm{m} / z$, 144.0575, found $m / z$ 144.0576.

1-Phenyl-2,3-butadien-1-ol (6a). Colorless oil ${ }^{1} \mathrm{H}$ NMR (400 MHz, $\left.\mathrm{CDCl}_{3}\right) \delta$ 7.42-7.25 (m, 5H), 5.45, (q, $J=3.6 \mathrm{~Hz}$, 1H), $5.27(\mathrm{~s}, 1 \mathrm{H}), 4.94-4.91(\mathrm{~m}, 2 \mathrm{H}) ;{ }^{13} \mathrm{C} \mathrm{NMR}\left(75 \mathrm{MHz}, \mathrm{CDCl}_{3}\right) \delta 206.7,142.2,128.3,127.7,125.9,95.1,78.2$, 71.9; IR (neat) 3373, 3030, 2891, 1955, 1494, 1454, 1002, $850 \mathrm{~cm}^{-1}$; HRMS (EI) calcd for $\mathrm{C}_{10} \mathrm{H}_{10} \mathrm{O}:\left(\mathrm{M}^{+}\right) \mathrm{m} / z$ 146.0732, found $m / z, 146.0726$.

1-Benzyloxy-1-phenyl-2,3-butadiene (6b). Yellow oil; ${ }^{1} \mathrm{H}$ NMR (400 MHz, $\left.\mathrm{CDCl}_{3}\right)$ 7.40-7.28 (m, $\left.10 \mathrm{H}\right), 5.35$ (q, J= $6.8 \mathrm{~Hz}, 1 \mathrm{H}), 4.94(\mathrm{~d}, J=7.6 \mathrm{~Hz}, 1 \mathrm{H}), 4.88-4.77(\mathrm{~m}, 2 \mathrm{H}), 4.58(\mathrm{q}, J=8.0 \mathrm{~Hz}, 2 \mathrm{H}) ;{ }^{13} \mathrm{C} \mathrm{NMR}\left(75 \mathrm{MHz}, \mathrm{CDCl}_{3}\right) \delta 208.50$, $141.18,138.08,128.26,128.20,127.65,127.58,127.40,126.50,92.71,79.27,76.46,70.18$; IR (neat) 2868, 1953, 1645, 1519, 1487, 1386, 1276, $1097 \mathrm{~cm}^{-1}$; HRMS (EI) calcd for $\mathrm{C}_{17} \mathrm{H}_{16} \mathrm{O}:\left(\mathrm{M}^{+}\right) \mathrm{m} / z$ 236.1201, found $\mathrm{m} / z, 236.1196$.

1-(4-Methoxyphenyl)-2,3-butadien-1-ol (6c). Colorless oil; ${ }^{1} \mathrm{H} \mathrm{NMR}\left(300 \mathrm{MHz}, \mathrm{CDCl}_{3}\right) \delta 7.32(\mathrm{~d}, J=8.7 \mathrm{~Hz}, 2 \mathrm{H})$, $6.88(\mathrm{~d}, J=8.7 \mathrm{~Hz}, 2 \mathrm{H}), 5.43(\mathrm{q}, J=6.6 \mathrm{~Hz}, 1 \mathrm{H}), 5.23(\mathrm{~s}, 1 \mathrm{H}), 4.93-4.89(\mathrm{~m}, 2 \mathrm{H}), 3.81,(\mathrm{~s}, 3 \mathrm{H}) ;{ }^{13} \mathrm{C} \mathrm{NMR}(75$ $\left.\mathrm{MHz}, \mathrm{CDCl}_{3}\right) \delta 206.7,159.0,134.9,127.2,113.7,95.2,78.0,71.5,55.2$; IR (neat) 3402, 2837, 1955, 1610, 1585, 1514, 1247, 1174, $1033 \mathrm{~cm}^{-1}$; HRMS (EI) calcd for $\mathrm{C}_{11} \mathrm{H}_{12} \mathrm{O}_{2}:\left(\mathrm{M}^{+}\right) \mathrm{m} / \mathrm{z}, 176.0838$, found $\mathrm{m} / \mathrm{z}, 176.0832$.

1-(3,5-Dimethoxyphenyl)- 2,3-butadien-1-ol (6d). Colorless oil; ${ }^{1} \mathrm{H}$ NMR $\left(400 \mathrm{MHz}, \mathrm{CDCl}_{3}\right) \quad \delta \quad 6.56(\mathrm{~d}, J=6.0 \mathrm{~Hz}$, $2 \mathrm{H}), 6.39(\mathrm{t}, J=6.4 \mathrm{~Hz}, 1 \mathrm{H}), 5.42(\mathrm{q}, J=6.4 \mathrm{~Hz}, 1 \mathrm{H}), 5.21(\mathrm{~s}, 1 \mathrm{H}), 4.95-4.93(\mathrm{~m}, 2 \mathrm{H}), 3.80(\mathrm{~s}, 6 \mathrm{H}) ;{ }^{13} \mathrm{C} \mathrm{NMR}(75$ $\left.\mathrm{MHz}, \mathrm{CDCl}_{3}\right) \delta 206.8,160.6,145.2,103.8,99.6,94.9,78.1,71.9,55.3$; IR (neat) $3421,2839,1955,1596,1458,1296$, 1205, $1060 \mathrm{~cm}^{-1}$; HRMS (EI) calcd for $\mathrm{C}_{12} \mathrm{H}_{14} \mathrm{O}_{3}:\left(\mathrm{M}^{+}\right) \mathrm{m} / \mathrm{z}, 206.0943$, found $\mathrm{m} / z$ 206.0937.

1,2-Nonadien-4-ol (6e). Colorless oil; ${ }^{1} \mathrm{H}$ NMR (400 MHz, $\mathrm{CDCl}_{3}$ ) $\delta 5.24$ (q, $\left.J=6.8 \mathrm{~Hz}, 1 \mathrm{H}\right), 4.86-4.84(\mathrm{~m}, 2 \mathrm{H})$, 4.19-4.14 (m, 1H), 1.58-1.54 (m, 2H), 1.43-1.25 (m, 6H), $0.89(\mathrm{t}, J=6.8 \mathrm{~Hz}, 3 \mathrm{H}) ;{ }^{13} \mathrm{C} \mathrm{NMR}\left(\mathrm{CDCl}_{3}\right) \delta 206.6,94.8,77.3$, 69.7, 37.5, 31.7, 25.1, 22.6, 14.1; IR(neat) 3336, 2931, 1955, 1508, 1458, 1022, $842 \mathrm{~cm}^{-1}$. Since the $\mathbf{M}^{+}$of $\mathbf{5 e}$ was not detected by the mass spectroscopy, the alcohol $\mathbf{6 e}$ was transformed into the benzoate form and assigned. Colorless oil; ${ }^{1} \mathrm{H}$ NMR $\left(400 \mathrm{MHz}, \mathrm{CDCl}_{3}\right) \delta 8.03(\mathrm{~d}, J=7.2 \mathrm{~Hz}, 2 \mathrm{H}), 7.53(\mathrm{t}, J=7.2 \mathrm{~Hz}, 1 \mathrm{H}), 7.42(\mathrm{t}, J=7.6 \mathrm{~Hz}, 2 \mathrm{H}), 5.27(\mathrm{t}, J=$ $5.1 \mathrm{~Hz}, 1 \mathrm{H}), 5.30(\mathrm{q}, J=5.1 \mathrm{~Hz}, 1 \mathrm{H}), 4.87-4.81(\mathrm{~m}, 2 \mathrm{H}), 1.83-1.73(\mathrm{~m}, 2 \mathrm{H}), 1.43-1.28(\mathrm{~m}, 6 \mathrm{H}), 0.86(\mathrm{t}, J=6.8 \mathrm{~Hz}, 3 \mathrm{H})$; ${ }^{13} \mathrm{C}$ NMR $\left(75 \mathrm{MHz}, \mathrm{CDCl}_{3}\right) \delta 206.6,94.8,77.3,69.7,37.5,31.7,25.1,22.6,14.1 ; \mathrm{MS}(\mathrm{EI}) \mathrm{m} / z 244\left(\mathrm{M}^{+}\right), 173$ $\left(\left[\mathrm{M}-\mathrm{C}_{5} \mathrm{H}_{11}\right]^{+}\right), 123\left([\mathrm{M}-\mathrm{OBz}]^{+}\right)$.

1-Allenylcyclohexanol (6f). Colorless oil; ${ }^{1} \mathrm{H} \mathrm{NMR}\left(400 \mathrm{MHz}, \mathrm{CDCl}_{3}\right) \delta 5.29(\mathrm{t}, J=6.8 \mathrm{~Hz}, 1 \mathrm{H}), 4.87(\mathrm{~d}, J=6.8 \mathrm{~Hz}$, 2H), 1.69-1.59 (m, 4H), $1.47(\mathrm{t}, J=5.2 \mathrm{~Hz}, 4 \mathrm{H}), 1.37-1.34(\mathrm{~m}, 2 \mathrm{H}) ;{ }^{13} \mathrm{C} \mathrm{NMR}\left(\mathrm{CDCl}_{3}\right) \delta 205.9,99.3,78.0,70.4,38.3$, 25.5, 22.6; IR(neat) $3367,2933,2856,1955,1508,952 \mathrm{~cm}^{-1}$; the $\mathbf{M}^{+}$of $\mathbf{6 f}$ was not detected by the mass spectroscopy.

\section{References}

1) Moreau, J-L.; Gaudemar, M. J. Organomet. Chem. 1976, 108, 159.

2) Takagi, K.; Tomita, I.; Endo, T. Macromolecules 1997, 30, 7386.

3) (a) Murahashi, S.-I.; Hirano, T.; Yano, T. J. Am. Chem. Soc. 1978, 100, 348. (b) Murahashi, S.-I.; Watanabe, T. J. Am. Chem. Soc. 1979, 101, 7430. 